\title{
Pulse nonlinear optical switching in plasmonic structures
}

\author{
José R. Salgueiro ${ }^{1,3, *}$ and Albert Ferrando ${ }^{2, * *}$ \\ ${ }^{1}$ Departamento de Física Aplicada, Universidade de Vigo, E. E. Aeronáutica e do Ezpazo, As Lagoas, s/n, 32004 Ourense, Spain. \\ ${ }^{2}$ Departament d'Òptica i Optometria i Ciències dela Visió, Universitat de València, Dr. Moliner, 50, Burjassot, 46100 Valencia, Spain.
}

\begin{abstract}
We study switching operation in a plasmonic coupler using fs-pulses. Simulations using the finitedifference time-domain method (FDTD) are carried out showing how the output changes as the pulse energy increases raising from zero to a maximum. Both cases of neglecting and realistic losses are considered in order to compare. The work is intended to explore the use of pulses for all-optical signal processing in a potentially interesting system for integrated photonics at the nanometric scale.
\end{abstract}

\section{Introduction}

Plasmonics has recently attracted a great interest because of the potential applications in nanophotonics, dramatically increasing the encapsulation possibilities of conventional integrated devices. In fact, integrated elements containing metals allow the light confinement to dimensions that are orders of magnitude lower than wavelength. This property together with the possibility of using nonlinear materials increases functionality allowing the design of integrated optical devices to carry out all-optical processing[1] in a nanometric scale. As nonlinear effects require high power density to trigger the suitable response it is quite common the use of pulsed sources where optical energy can be concentrated in a short time (of the order of femtoseconds). This makes necessary a specific modeling for this kind of systems as they behave generally in quite a different way respect to the case of using continuous beams since temporal and dispersive effects come into play. In the past some studies with pulses in plasmonic systems were carried out, focused on surface plasmon polariton (SPP) dynamics[2, 3] and slot waveguides [4, 5].

In this work we demonstrate the possibility of accomplishing switching in plasmonic directional couplers using short pulses, based on the total energy of the input pulse. A similar system was already studied for a CW regime[6], using in that case optical power to trigger the change of state. In the following, details of the study as it concerns modeling and results are presented.

\section{System modeling}

A system of two slot waveguides separated by a short distance was modeled using the FDTD method[7]. Both cores are dielectric presenting a nonlinear third-order Kerr response while claddings are supposed to be linear and

\footnotetext{
*e-mail: jrs@uvigo.es

**e-mail: Albert.Ferrando@uv.es
}

metallic. Although a simple instantaneous-response model was used for the nonlinear cores, the method leaves the door open to a future inclusion of more elaborated or specific models, dependent on the pulse duration as for instance the third order Raman dispersion. Anyway, the pulsed character of the modeled beams is important as metals are strongly dispersive and have to be described by a model that properly takes into account such dispersion. In order to compare realistic results including losses to the case for which loss is absent, we modeled the metal
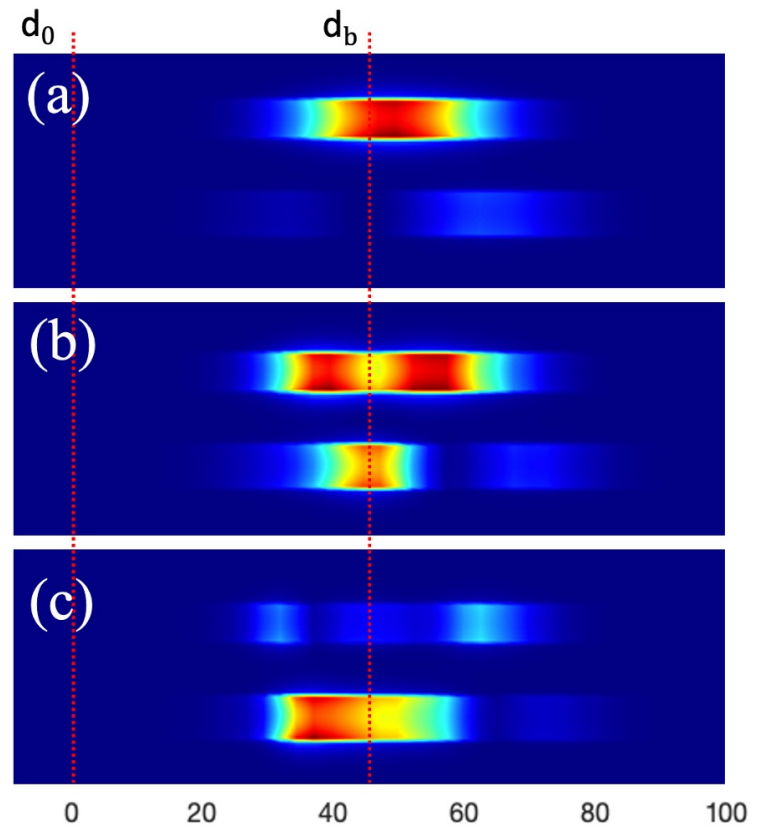

Figure 1. Three simulations for three input pulses of different energy launched into the low waveguide of the coupler and for the same propagation time. Input powers are (normalized units) $W_{i n}=0.43(\mathrm{~A}), W_{i n}=3.45(\mathrm{~B})$ and $W_{i n}=6.03(\mathrm{C})$. Vertical lines mark the waveguide entrance at position $d_{0}=0$ and the output located at a beat length $d_{b}$. 
using a classical Drude method which characterizes the medium by a plasma frequency $\omega_{p}$ and an electronic collision frequency $\Gamma$ (which is identically zero when losses are neglected)[8]. Anyway, it would be possible the use of more realistic models at optical frequencies as the modified Debye method[9], though in this case it would not be possible to isolate losses from the set of parameters and would had to be necessarily taken into account.

The calculation domain was surrounded by perfect matching layer zones (PML) to get rid of all outgoing radiation. Also, a source based on the total-field/scatteredfield technique was implemented to generate a pulse at the entrance of one of the waveguides presenting a Gaussian transversal shape and also Gaussian temporal shape. The TM-polarization was used considering two electric components $\left(E_{x}\right.$ and $\left.E_{z}\right)$ in the simulation plane and a magnetic component $\left(H_{y}\right)$ orthogonal to the plane.

\section{Results}

For the simulations we chose silver as the metal for the cladding, characterized by the parameters $\omega_{p}=1.15 \times 10^{16}$ $\mathrm{Hz}$ and $\Gamma=9 \times 10^{13} \mathrm{~Hz}$ at a wavelength of $\lambda_{0}=800 \mathrm{~nm}$. Spatial coordinates were normalized by the wavenumber $k_{0}=2 \pi / \lambda_{0}$. In such normalized units we took the width of both cores as well as the separation between them as 0.5 (corresponding to about $65 \mathrm{~nm}$ ). Pulses where generated with the same width and a time duration of $115 \mathrm{fs}$ and launched into one of the cores (first core). Power at a particular position (propagation length) was measured integrating the longitudinal $(z)$ component of the Poynting vector $(\vec{S}=\vec{E} \times \vec{H})$ spatially on the transversal direction and also over time to obtain the total energy of the propagating pulse:

$$
W(z)=\int_{t} \int_{x}[\vec{S}(x, z) \cdot \hat{z}] \mathrm{d} x \mathrm{~d} t .
$$

The first simulation was in a linear regime, intended to determine the beat length for the coupler, i. e. the propagation distance necessary to produce the maximum transfer

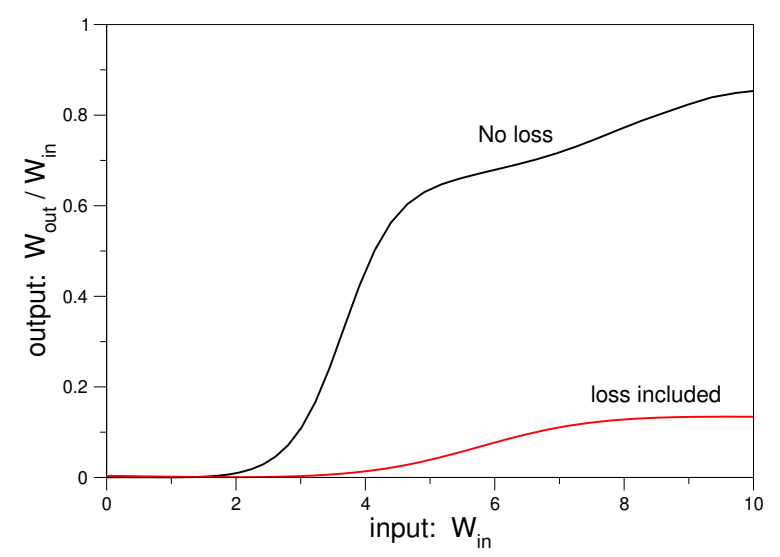

Figure 2. Switching curve: ratio of output and input pulse energy plotted against input pulse energy for both cases of neglected loss and realistic loss. of the pulse energy to the second core. This was measured as $d_{b} \approx 45(5.73 \mu \mathrm{m})$. Next, a set of simulations where carried out, launching similar pulses with increasing total energy but same duration. In figure 1 it is plotted the power flux (time average of the pointing vector) for three different simulations correspondent to a linear (a), intermediate (b) and full nonlinear (c) regimes. In (a) power is completely transferred to the second core after a distance $d_{b}$ producing an almost null signal at the first core output. In (b) only a fraction of energy is transferred while in (c) most of the energy remains in the first core, producing a maximum signal at the first core output.

The switching operation is illustrated in figure 2 for both cases of losses neglected and losses included. The plot represents normalized output energy against input energy. For low input signal the output is almost null as all the energy transfers to the second core. At some point there is a fast rise of the output signal (switching) to maximum values. When losses are taken into account switching is still possible but there are two important differences. On one hand output energy is quite lower (as part was converted into heat by Joule effect). On the other hand there is a loss of efficiency as the slope of the curve decreases in such a way that the rising signal extends over a double range of energy (horizontal axis) respect to the case of neglecting losses. This behavior was already observed for $\mathrm{CW}$ beams [6] and even solutions were proposed to improve performance[10].

The authors wish to thank the government of Spain and the local government of Galicia for the economical support under contracts FIS2017-83762-P and ED431B2018/57-GPC respectively. Financial support from Spanish MINECO through Project No. TEC2017-86102-C2-1-R is gratefully acknowledged.

\section{References}

[1] N.C. Panoiu, W.E.I. Sha, D.Y. Lei, G.C. Li, Journal of Optics 20, 083001 (2018)

[2] Z.L. Sámson, P. Horak, K.F. MacDonald, N.I. Zheludev, Opt. Lett. 36, 250 (2011)

[3] A. Baron, T.B. Hoang, C. Fang, M.H. Mikkelsen, D.R. Smith, Phys. Rev. B 91, 195412 (2015)

[4] A. Pusch, I.V. Shadrivov, O. Hess, Y.S. Kivshar, Opt. Express 21, 1121 (2013)

[5] O. Lysenko, M. Bache, N. Olivier, A.V. Zayats, A. Lavrinenko, ACS Photonics 3, 2324 (2020)

[6] J.R. Salgueiro, Y.S. Kivshar, Appl. Phys. Lett. 97, 081106 (2010)

[7] A. Taflove, Computational electrodynamics, the finite difference time domain (Artech House Publishers, Norwood, Massachussets, 2005)

[8] S.A. Cummer, IEEE Trans. Antennas Propagat. 45, 392 (1997)

[9] H. Gai, J. Wang, Q. Tian, Appl. Opt. 46, 2229 (2007)

[10] J.R. Salgueiro, Y. Kivshar, Opt. Expr. 20, 9403 (2012) 